\title{
Impaired Performance on the Reading the Mind in the Eyes Test in First-Episode Psychosis and Clinical High Risk for Psychosis
}

\author{
Seo Woo Kim ${ }^{1}$, Sun-Young Moon ${ }^{2,3}$, Wu Jeong Hwang', Silvia Kyungjin Lho, ${ }^{2,3}$ \\ Sanghoon $\mathrm{Oh}^{2,3}$, Tae Young Lee ${ }^{4,5}$, Minah $\mathrm{Kim}^{2,3}$, and Jun Soo Kwon ${ }^{2,3,5}$ \\ 1'Department of Brain and Cognitive Sciences, Seoul National University College of Natural Sciences, Seoul, Republic of Korea \\ ${ }^{2}$ Department of Psychiatry, Seoul National University College of Medicine, Seoul, Republic of Korea \\ ${ }^{3}$ Department of Neuropsychiatry, Seoul National University Hospital, Seoul, Republic of Korea \\ ${ }^{4}$ Department of Neuropsychiatry, Pusan National University Yangsan Hospital, Yangsan, Republic of Korea \\ ${ }^{5}$ Institute of Human Behavioral Medicine, SNU-MRC, Seoul, Republic of Korea
}

Objective Although previous studies have reported impaired performance in the reading the mind in the eyes test (RMET), which measures complex emotion recognition abilities, in patients with schizophrenia, reports regarding individuals at clinical high risk (CHR) for psychosis have been inconsistent, mainly due to the interacting confounding effects of general cognitive abilities and age. We compared RMET performances across first-episode psychosis (FEP) patients, CHR individuals, and healthy controls (HCs) while controlling for the effects of both general cognitive abilities and age.

Methods A total of $25 \mathrm{FEP}, 41 \mathrm{CHR}$, and $44 \mathrm{HC}$ subjects matched for age participated in this study. RMET performance scores were compared across the groups using analysis of variance with sex and intelligence quotient as covariates. Exploratory Pearson's correlation analyses were performed to reveal the potential relationships of RMET scores with clinical symptom severity in the FEP and CHR groups.

Results RMET performance scores were significantly lower among FEP and CHR participants than among HCs. FEP patients and CHR subjects showed comparable RMET performance scores. RMET scores were negatively correlated with Positive and Negative Syndrome Scale (PANSS) positive symptom subscale scores in the FEP patients. No significant correlation was identified between RMET scores and other clinical scale scores.

Conclusion Impaired RMET performance is present from the risk stage of psychosis, which might be related to positive symptom severity in early psychosis. Longitudinal studies are necessary to confirm the stability of complex emotion recognition impairments and their relationship with social functioning in early psychosis patients.

Psychiatry Investig 2020;17(12):1200-1206

Key Words Clinical high risk for psychosis, First-episode psychosis, Theory of mind, Reading the mind in the eyes test, Schizophrenia.

\section{INTRODUCTION}

Social dysfunction affects the quality of life of patients with schizophrenia and has been associated with a poor treatment response and prognosis in early psychosis patients. ${ }^{1,2}$ Therefore, researchers have tried to investigate factors that can explain social dysfunction in schizophrenia patients to improve

Received: July 8, 2020 Revised: September 11, 2020

Accepted: September 23, 2020

$\triangle$ Correspondence: Minah Kim, MD, PhD

Department of Neuropsychiatry, Seoul National University Hospital, 101 Daehak-no, Jongno-gu, Seoul 03080, Republic of Korea

Tel: +82-2-2072-2972, Fax: +82-2-747-9063, E-mail: verte82@snu.ac.kr

(a) This is an Open Access article distributed under the terms of the Creative Commons Attribution Non-Commercial License (https://creativecommons.org/licenses/by$\mathrm{nc} / 4.0$ ) which permits unrestricted non-commercial use, distribution, and reproduction in any medium, provided the original work is properly cited. the quality of life and prognosis of patients with psychotic disorders. Theory of mind (ToM), which refers to the ability to recognize the beliefs, intentions, and emotion of others, has been identified as the most important cognitive domain that best explains the social dysfunctions observed in schizophrenia patients. ${ }^{3-5}$ In addition, impaired ToM has been found to be related to and may be a risk factor for psychotic symptoms such as paranoia and disorganization..$^{6,7}$

Tasks to measure ToM can be divided into two major categories-reasoning and complex emotion recognition. ${ }^{5}$ In reasoning ToM tasks, such as the hinting task, the story task, and the false belief task, subjects are instructed to infer others' beliefs or intentions by integrating contextual information by means of a comprehensive set of semantic abilities. ${ }^{8,9}$ Impaired performance in reasoning ToM tasks has been consistently re- 
ported in various clinical stages of psychotic disorders ranging from chronic schizophrenia to first-episode psychosis (FEP) and clinical high risk (CHR) for psychosis. ${ }^{3,10,11}$ However, nonsocial cognitive components such as the intelligence quotient (IQ), working memory, and executive function might compensate for actual reasoning ToM performance, especially in CHR subjects. ${ }^{12-14}$

The representative task in the complex emotion recognition category of ToM is the reading the mind in the eyes test (RMET), which assesses complex emotion recognition abilities to perceive others' emotions through the eyes. ${ }^{8,15}$ Contrary to basic emotions (e.g., joy, sadness, fear, anger, disgust), complex emotions (e.g., shame, pride) are more subtle and essential to forming sophisticated social relationships throughout life. ${ }^{16,17}$ Although both cognitive and perceptual processes occur during RMET performance, ${ }^{5,18}$ perceptive aspects are thought to play a vital role because RMET performance mainly depends on the ability to perceive imminent, observable information from facial expressions. ${ }^{8,9}$ Therefore, RMET performance may be less affected by general cognitive abilities and more representative of real-world social cognitive functioning in psychosis patients. ${ }^{8,19,20}$

Impaired RMET performance has been consistently reported in chronic schizophrenia and FEP patients. ${ }^{3,10,21}$ However, previous study results regarding CHR individuals have not reached a clear conclusion in that three studies that matched IQ across the groups reported intact RMET performance, ${ }^{22-24}$ whereas two studies from the same research group that did not assess IQ scores reported impairments in CHR subjects. ${ }^{12,25}$ Such inconsistencies may be explained by the fact that social cognitive performance in CHR individuals is affected by two important interacting confounders, namely, general cognitive ability and age. ${ }^{26,27}$ The influence of general cognitive abilities on social cognitive performance has been suggested to be larger during adolescence than during adulthood. ${ }^{28-30}$ However, previous studies comparing RMET performance between CHR subjects, who are mostly in adolescence and early adulthood when general cognitive abilities affect social cognition, and schizophrenia patients and healthy controls (HCs), who are mostly in adulthood when general cognitive abilities have less effect on social cognition, did not simultaneously control for age and IQ. ${ }^{12,22-25}$ Furthermore, little is known about the associations between RMET performance and psychotic symptom severity in early psychosis patients. Two previous studies reported no significant relationship between RMET performance and symptom severity in FEP patients ${ }^{31}$ and CHR subjects, ${ }^{22}$ whereas another study reported an association between RMET performance and psychotic symptom severity in helpseeking individuals. ${ }^{32}$

In the current study, we compared RMET performance be- tween age-matched patients with FEP, subjects at CHR for psychosis, and HCs to confirm whether impairments in complex emotion recognition abilities measured by the RMET are present from the CHR stage after controlling for the interacting effects of general cognitive abilities and age. We hypothesized that age-matched FEP and CHR participants would show comparably poorer RMET performance than HCs when IQ was used as a covariate to control for the effect of general cognitive abilities. In addition, to reveal possible relationships between complex emotion recognition abilities and psychotic symptoms in these clinical populations, exploratory correlation analyses between RMET performance and symptom severity were performed in the CHR and FEP groups.

\section{METHODS}

\section{Participants and clinical assessments}

A total of $25 \mathrm{FEP}$ patients, $41 \mathrm{CHR}$ individuals, and $45 \mathrm{HCs}$ matched for age participated in this study (the age range of all three groups was 18 to 32 years). FEP and CHR participants were recruited from the prospective and longitudinal highrisk cohort study conducted at the Seoul Youth Clinic (http:// www.youthclinic.org) in Seoul National University Hospital (SNUH). ${ }^{33} \mathrm{HCs}$ were recruited through an Internet advertisement and screened for axis I diagnoses with the SCID-I NonPatient Edition (SCID-NP). HCs with a family history of psychotic disorder were excluded from the study. The abbreviated version of the Korean-Wechsler Adult Intelligence Scale was used to measure IQ in all participants. ${ }^{34}$ The common exclusion criteria included intellectual disability (IQ $<70$ ), a history of substance use disorder, neurological disease, head trauma with loss of consciousness, seizure, or any other significant medical illnesses.

FEP patients were defined when they met the diagnostic criteria for schizophreniform disorder, schizophrenia, or schizoaffective disorder according to the Diagnostic and Statistical Manual of Mental Disorders, Fourth Edition (DSM-IV) and the duration of illness was less than 2 years. The Positive and Negative Symptom Scale (PANSS) was administered to assess psychotic symptom severity. The CHR subjects were assessed using the validated Korean version of the Structured Interview for Prodromal Symptoms (SIPS). ${ }^{35,36}$ Prodromal status was confirmed if the participants met at least one of the following three criteria: 1) attenuated positive symptoms (APSs), 2) the presence of brief intermittent psychotic symptoms (BIPSs), and 3) a genetic risk with deterioration (GRD). The severity of prodromal psychotic symptoms was assessed using the Scale of Prodromal Symptoms (SOPS) ${ }^{35,36}$ Intensive clinical interviews were conducted by experienced psychiatrists using the Structured Clinical Interview for the Diagnostic and Statisti- 
cal Manual of Mental Disorders, Fourth Edition Axis I disorders (SCID-I) to identify past and current psychiatric illnesses. To define general functional status, the global assessment of functioning (GAF) was administered to both the CHR and FEP groups. Medication prescriptions at the time of enrollment were reviewed through electronic medical records. At the time of assessment, $88 \%(n=22)$ of FEP patients were receiving atypical antipsychotic medications, a mood stabilizer was prescribed for 1 patient, antidepressants were prescribed for 3 patients, and anxiolytics were prescribed for 14 patients. At the time of enrollment, $17 \%(n=7)$ of the CHR subjects were receiving antipsychotic medications, mood stabilizers were prescribed for 3 patients, antidepressants were prescribed for 11 patients, and anxiolytics were prescribed for 12 patients. The dose of antipsychotic medication prescribed was calculated as the mean olanzapine equivalent dose. ${ }^{37}$ The mean daily dose for the FEP patients calculated by the olanzapine equivalent dose was $11.9 \pm 10.3 \mathrm{mg}$, and that for the CHR subjects was $0.6 \pm 2.2 \mathrm{mg}$.

This study was conducted according to the Declaration of Helsinki and was approved by the Institutional Review Board of SNUH (IRB no. H-1912-108-108). Each subject received a complete description of the study and provided written informed consent before participation in the previous prospective cohort study (IRB no. H-1201-008-392). For the minors who participated in this study, informed consent was obtained from both the participants and their parents.

\section{Reading the mind in the eyes test}

The validated Korean version of the RMET was used to assess complex emotion recognition abilities in all participants, ${ }^{38}$ which included 36 photographs of the eyes of Korean actors and actresses from movies with various expressions. All photos in the study were black and white, and each photo was $15 \times 6 \mathrm{~cm}$ in size (Figure 1). For each item, the participants were asked to choose one of four words that best described the ex-

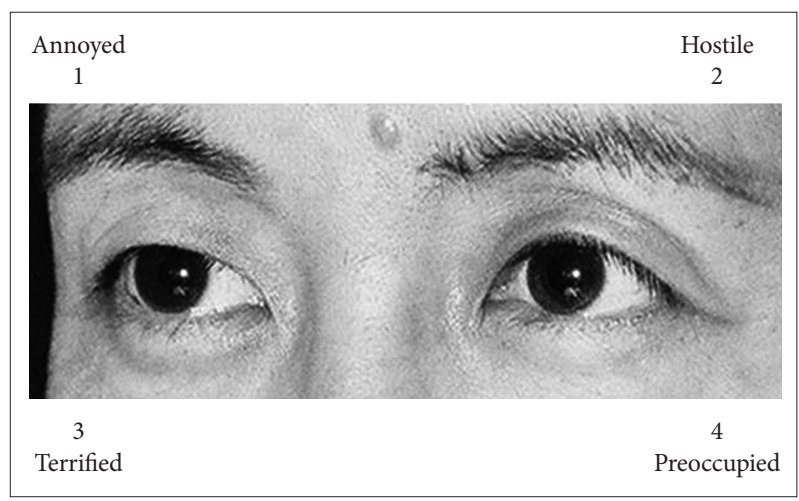

Figure 1. Example from the Korean version of the reading the mind in the eyes test (RMET). pression in the eyes. No time limits were imposed, and all emotional words conformed to the original task described by Baron-Cohen et al. ${ }^{15}$ RMET performance scores were calculated as the sum of the number of correct answers (i.e., a higher score depicts better performance on the RMET).

\section{Statistical analysis}

The statistical analyses were performed using SPSS v.25.0 (IBM Corp., Armonk, NY, USA). Statistical significance was set at $\mathrm{p}<0.05$. Demographic characteristics were compared using analysis of variance (ANOVA) across the FEP, CHR, and $\mathrm{HC}$ groups for continuous variables. Chi-square tests were used for categorical variables. Clinical characteristics, including symptom severity and the olanzapine equivalent dose of antipsychotics, were compared using independent samples ttests in the FEP and CHR groups. Univariate analysis of covariance (ANCOVA) was conducted to examine group differences in RMET performance. The covariate included in the ANCOVA was sex, which was significantly different across the groups. IQ was also used as a covariate because the RMET is known to have a correlation with general intelligence in the normal population ${ }^{39}$ and to control the possible confounding effect of general cognitive abilities in youth with early psychosis. ${ }^{26}$ Bonferroni corrections were applied for post hoc analyses. Exploratory Pearson's correlation analyses were performed to reveal the potential relationships between RMET performance scores and symptom severity and general functional status in the FEP and CHR groups.

\section{RESULTS}

Demographic and clinical characteristics of the subjects

Table 1 summarizes the demographic and clinical characteristics of the subjects. A significant group difference in sex was noted, with more females in the FEP group than in the CHR and HC groups $\left(\chi^{2}=11.871, p=0.003\right)$. No significant difference in age or education years was found across all groups. General functional status measured by the GAF did not differ between the FEP and CHR participants $(\mathrm{t}=0.816, \mathrm{p}=0.417)$. In addition, the FEP patients received a larger olanzapine equivalent dose of antipsychotic medications than the CHR subjects $(\mathrm{t}=6.793, \mathrm{p}<0.001)$.

\section{Group comparison of RMET performance}

Group comparison results for RMET performance scores are presented in Table 2 and Figure 2. ANOVA with sex and IQ as covariates revealed a significant group difference in RMET performance scores across the $\mathrm{FEP}, \mathrm{CHR}$, and $\mathrm{HC}$ groups $\left(\mathrm{F}_{2,105}=5.174, \mathrm{p}=0.007\right)$. Post hoc Bonferroni correction showed that both FEP patients $(\mathrm{p}=0.013)$ and CHR individuals $(\mathrm{p}=$ 
Table 1. Demographic and clinical characteristics of patients with first-episode psychosis (FEP), subjects at clinical high risk (CHR) for psychosis, and healthy controls (HCs)

\begin{tabular}{|c|c|c|c|c|c|c|c|c|}
\hline & \multirow{2}{*}{$\begin{array}{c}\text { FEP } \\
(\mathrm{N}=25)\end{array}$} & \multirow{2}{*}{$\begin{array}{c}\mathrm{CHR} \\
(\mathrm{N}=41)\end{array}$} & \multirow{2}{*}{$\begin{array}{c}\mathrm{HC} \\
(\mathrm{N}=44)\end{array}$} & \multicolumn{2}{|c|}{ Statistical analysis $^{\mathrm{a}}$} & \multicolumn{3}{|c|}{ Post hoc analysis ${ }^{b}$} \\
\hline & & & & $\mathrm{F}$ or $\mathrm{T}$ or $\chi^{2}$ & $\mathrm{p}$ & FEP vs. CHR & FEP vs. HC & CHR vs. HC \\
\hline Age (years) & $22.9 \pm 3.9$ & $21.2 \pm 3.1$ & $21.8 \pm 3.4$ & 1.684 & 0.190 & 0.208 & 0.741 & 1.000 \\
\hline Sex (male/female) & $10 / 15$ & $31 / 10$ & $34 / 10$ & 11.871 & $0.003^{\dagger}$ & - & - & - \\
\hline IQ & $105.3 \pm 15.9$ & $104.0 \pm 12.2$ & $109.0 \pm 11.0$ & 1.673 & 0.193 & 1.000 & 0.794 & 0.233 \\
\hline Education (years) & $14.2 \pm 2.1$ & $13.1 \pm 1.6$ & $13.9 \pm 1.8$ & 3.234 & $0.043^{*}$ & 0.060 & 1.000 & 0.181 \\
\hline \multicolumn{9}{|l|}{ PANSS } \\
\hline Positive symptoms & $13.6 \pm 5.5$ & - & - & - & - & - & - & - \\
\hline Negative symptoms & $15.0 \pm 7.0$ & - & - & - & - & - & - & - \\
\hline General symptoms & $29.0 \pm 10.3$ & - & - & - & - & - & - & - \\
\hline \multicolumn{9}{|l|}{ SOPS } \\
\hline Positive symptoms & - & $11.6 \pm 4.0$ & - & - & - & - & - & - \\
\hline Negative symptoms & - & $14.3 \pm 6.3$ & - & - & - & - & - & - \\
\hline Disorganization & - & $4.5 \pm 3.6$ & - & - & - & - & - & - \\
\hline General symptoms & - & $7.6 \pm 4.1$ & - & - & - & - & - & - \\
\hline GAF & $54.8 \pm 16.2$ & $52.2 \pm 9.1$ & - & 0.816 & 0.417 & - & - & - \\
\hline Antipsychotics dose $\mathrm{c}^{\mathrm{c}}$ & $11.9 \pm 10.3$ & $0.6 \pm 2.2$ & - & 6.793 & $<0.001^{\dagger}$ & & & \\
\hline
\end{tabular}

Data are given as the mean \pm standard deviation. analysis of variance, independent $t$ test or Welch's $t$ test if the variances were not equal, $\chi^{2}$ analysis or Fisher's exact test for categorical data, ${ }^{b}$ Post hoc Bonferroni correction analysis, 'olanzapine equivalent dose of antipsychotics prescribed at the time of enrollment. *the mean difference is significant at the 0.05 level, the mean difference is significant at the 0.005 level. IQ: intelligence quotient, PANSS: Positive and Negative Syndrome Scale, SOPS: Scale of Prodromal Symptoms, GAF: global assessment of functioning

Table 2. Results from the reading the mind in the eyes test (RMET) across the three groups

\begin{tabular}{|c|c|c|c|c|c|c|c|c|}
\hline & \multirow{2}{*}{$\begin{array}{c}\text { FEP } \\
(\mathrm{N}=25)\end{array}$} & \multirow{2}{*}{$\begin{array}{c}\text { CHR } \\
(\mathrm{N}=41)\end{array}$} & \multirow{2}{*}{$\begin{array}{c}\mathrm{HC} \\
(\mathrm{N}=44)\end{array}$} & \multicolumn{2}{|c|}{ Statistical analysis ${ }^{\mathrm{a}}$} & \multicolumn{3}{|c|}{ Post hoc analysis ${ }^{\mathrm{b}}$} \\
\hline & & & & $\mathrm{F}$ & $\mathrm{p}$ & FEP vs. CHR & FEP vs. HC & CHR vs. HC \\
\hline RMET scores & $22.8 \pm 5.2$ & $23.4 \pm 3.8$ & $25.8 \pm 3.0$ & 5.174 & $0.007^{*}$ & 1.000 & $0.013^{*}$ & $0.049^{*}$ \\
\hline
\end{tabular}

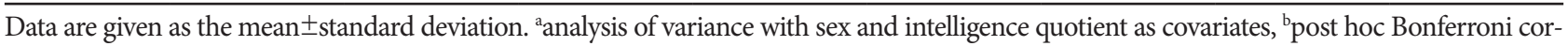
rection analysis. *the mean difference is significant at the 0.05 level. FEP: first-episode psychosis, CHR: clinical high risk, HC: healthy control

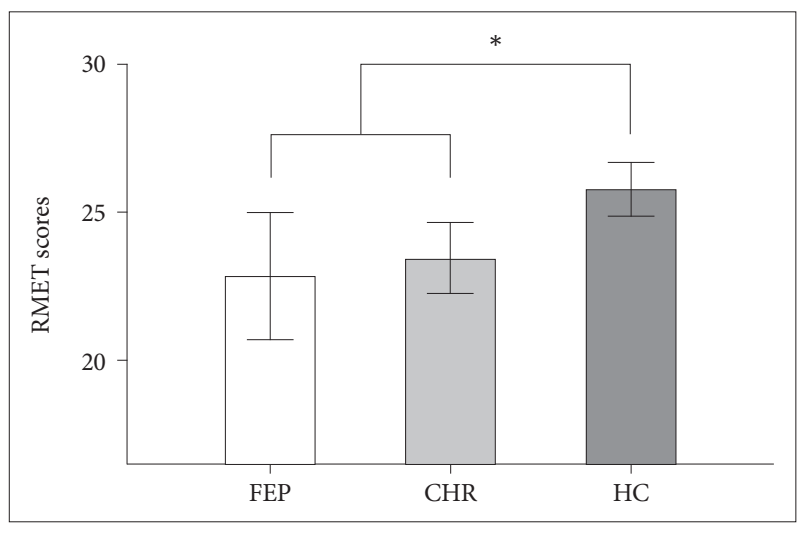

Figure 2. Group comparison of the reading the mind in the eyes test (RMET) performance across the first-episode psychosis (FEP), clinical high risk (CHR) for psychosis, and healthy control (HC) groups. The horizontal lines for each group indicate the means, and the vertical lines for each group indicate the 95\% confidence intervals. *indicates that the mean difference is significant at the 0.05 level.
0.049) had significantly lower RMET performance scores than HCs.

\section{Exploratory correlation analysis results}

Exploratory Pearson's correlation analyses were conducted to investigate the potential relationships between RMET performance scores and clinical symptom severity in the FEP and CHR groups. These results are summarized in Table 3. PANSS positive symptom subscale scores were negatively correlated with RMET performance scores $(r=-0.419, \mathrm{p}=0.037)$ in the FEP patients (Figure 3 ). In the CHR group, no significant correlation was found between RMET performance scores and SOPS subscale scores. Furthermore, we found no significant correlation between GAF scores and RMET performance scores in both clinical groups (FEP, $\mathrm{p}=0.141$; $\mathrm{CHR}, \mathrm{p}=0.306$ ). 
Table 3. Exploratory Pearson's correlation analysis between reading the mind in the eyes test (RMET) scores and symptom severity

\begin{tabular}{clcl}
\hline Group & \multicolumn{1}{c}{ Clinical assessment } & $\mathrm{r}$ & $\mathrm{p}$ \\
\hline \multirow{2}{*}{ FEP } & PANSS positive symptoms & -0.419 & $0.037^{*}$ \\
& PANSS negative symptoms & -0.377 & 0.064 \\
& PANSS general symptoms & -0.325 & 0.113 \\
CHR & SOPS positive symptoms & -0.012 & 0.939 \\
& SOPS negative symptoms & -0.300 & 0.056 \\
& SOPS disorganization & -0.065 & 0.685 \\
& SOPS general symptoms & -0.147 & 0.358 \\
\hline
\end{tabular}

*the correlation is significant at the 0.05 level. FEP: first-episode psychosis, CHR: clinical high risk, PANSS: Positive and Negative Syndrome Scale, SOPS: Scale of Prodromal Symptoms

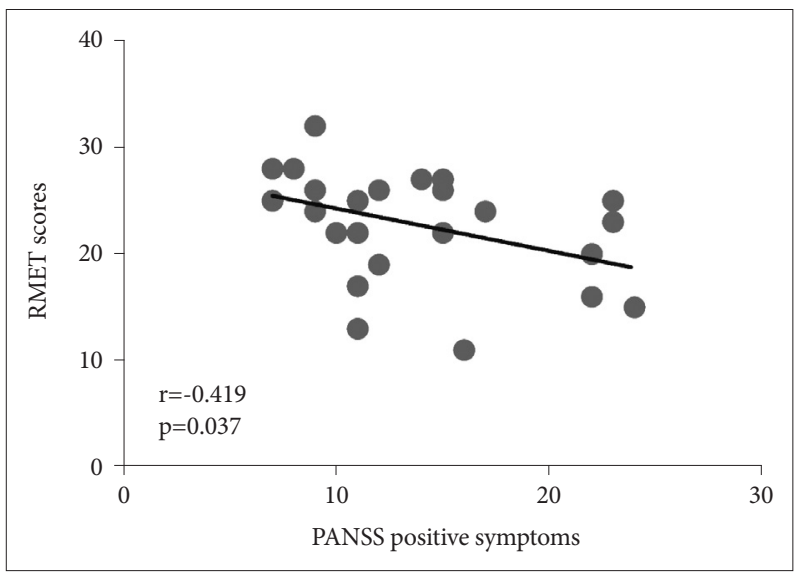

Figure 3. Correlation analysis between reading the mind in the eyes test (RMET) performance scores and Positive and Negative Syndrome Scale (PANSS) positive symptom subscale scores in first-episode psychosis (FEP) patients. The total number of dots in this figure is 24 even though the total number of FEP patients is 25 because of two overlapping dots with the same RMET score (28) and PANSS positive score (7).

\section{DISCUSSION}

The RMET is a useful tool to measure complex emotion recognition, with its study characteristics being intuitive (i.e., requiring little semantic or reasoning abilities) and engaging meaningful features of interpersonal relationships. ${ }^{15}$ While evidence for impaired RMET performance in chronic schizophrenia and FEP patients is quite solid, ${ }^{9,10,21}$ the existing literature regarding CHR individuals has reported inconsistent results. ${ }^{12,22-25}$ These inconsistencies may be partly due to the interacting confounding effects of general cognitive abilities and age on RMET performance, ${ }^{26,27}$ which were not controlled in previous studies. To address this issue, we examined RMET performance in age-matched FEP, CHR, and HC groups while controlling for IQ and found a similar degree of impaired performance on the RMET in both the FEP and CHR groups compared to the HC group. In addition, exploratory correlation analysis re- vealed that RMET performance in the FEP patients was negatively correlated with positive symptom severity as measured by the PANSS.

In the current study, complex emotion recognition abilities were impaired in age-matched FEP and CHR participants compared to HCs when the analyses were adjusted for sex and IQ. Our results are different from those of previous studies reporting intact RMET performance in CHR individuals. ${ }^{22-24} \mathrm{~A}$ wide range of participant ages in the three prior studies might have disguised true disabilities in complex emotion recognition in the CHR group. In fact, social cognitive abilities, including complex emotion recognition, develop rapidly during adolescence and early adulthood, and the relevance of general cognition to social cognition changes during the course of development, being greater in young individuals than in adults. ${ }^{17,27,30,40}$ Because CHR and FEP are largely distributed across adolescence and adulthood, comparing RMET performance across age-matched participants while controlling for the effect of IQ might have enabled us to observe obvious impairments in complex emotion recognition abilities in CHR subjects.

Notably, the RMET performance impairments observed in the CHR individuals were comparable to those in the FEP patients after controlling for IQ. The nature of the RMET, which requires instant perceptive abilities (i.e., eye gaze and face recognition) and less semantic reasoning, might have contributed to the similarly impaired RMET performance in the CHR subjects and FEP patients. ${ }^{5,8,9}$ Previous studies have demonstrated that $\mathrm{CHR}$ individuals have dysfunctional visual and facial perception abilities comparable to those of FEP and chronic schizophrenia patients. ${ }^{41,42}$ Therefore, the comparably impaired RMET performance in CHR and FEP individuals might represent significant perceptual disturbances not fully offset by general cognitive abilities such as IQ. In addition, this result indicates that traces of social dysfunction as measured by the RMET are present from the psychosis risk stage and may not be a result of the onset of psychosis. Therefore, the RMET can be useful tool both for early detection of individuals at CHR for psychosis and the development of a treatment tool for social cognitive functioning.

We found a significant negative association between RMET performance and positive symptom severity in the FEP patients, which is in line with the mechanism of sustained psychotic symptoms reported by a previous study suggesting that distortion in emotion recognition may contribute to the maintenance of faulty attribution or delusions. ${ }^{23}$ On the other hand, Vohs et al. ${ }^{43}$ reported a significant association between RMET performance and negative symptoms in FEP patients, which was not found in our study. However, according to the study by Guastella et al. ${ }^{32}$ while RMET performance was related to both positive and negative symptoms, it significantly predict- 
ed only positive symptom severity when IQ and other neurocognitive abilities, such as verbal learning and sustained attention, were considered. In our study, no significant difference in IQ was found across the groups, indicating that the general intelligence of our FEP group was relatively intact, which may account for the effaced association between negative symptoms and RMET scores. In addition, since our FEP group patients were mostly stabilized after acute treatment, we can infer that targeting remnant positive symptoms may be more specifically useful for improving complex emotion recognition rather than other symptom dimensions in these patients.

Several limitations exist in this study. First, a relatively small number of participants were included in the FEP group compared to the CHR and HC groups to match age. Second, significantly more females were included in the FEP group than in the other two groups, and most of the patients with FEP were taking antipsychotic medications at the time of participation in the experiment. However, previous meta-analyses demonstrated that sex and antipsychotic medication were not factors explaining impaired social cognition in schizophrenia patients. ${ }^{13,21}$ Although we used sex as a covariate in the group comparisons, the results of the current study should be interpreted with caution considering the potential confounding effects of sex and antipsychotic medication on RMET performance. Third, because of the cross-sectional study design, we could not address longitudinal changes in RMET performance across the different stages of early psychosis.

In conclusion, this study first demonstrated similarly impaired complex emotion recognition abilities in the age-matched FEP and CHR groups after controlling for the potential confounding effect of IQ compared to the HC group. In addition, the impairment in RMET performance did not differ between the CHR and FEP groups, suggesting that substantial impairment in recognizing complex emotions through the eyes begins to emerge at the prodromal stage of illness. Furthermore, RMET performance in the FEP group was specifically associated with the positive symptom dimension, suggesting that appropriate treatment to allay positive symptoms may facilitate the perception of subtle interpersonal emotions in this group. Identification and correction of individual attribution errors through individualized therapy (e.g., cognitive therapy) may also be helpful. Future longitudinal studies with age-, sex-, and IQ-matched, medication-naïve participants are warranted to confirm the current study results.

\section{Acknowledgments}

Funding was provided by the Basic Science Research Program through the National Research Foundation of Korea (NRF) funded by the Ministry of Science, ICT \& Future Planning (Grant nos. 2019R1C1C1002457 and 2019R1A2B5B03100844).

\section{Conflicts of Interest}

The authors have no potential conflicts of interest to disclose.

\section{Author Contributions}

Conceptualization: Minah Kim, Seo Woo Kim, Jun Soo Kwon. Data curation: Sun-Young Moon, Wu Jeong Hwang, Silvia Kyungjin Lho, Sanghoon Oh, Tae Young Lee. Formal analysis: Seo Woo Kim, Minah Kim. Funding acquisition: Minah Kim, Jun Soo Kwon. Investigation: all authors. Methodology: Seo Woo Kim, Minah Kim. Project administration: Minah Kim. Resources: Minah Kim, Jun Soo Kwon. Software: Seo Woo Kim. Supervision: Minah Kim. Validation: Minah Kim, Tae Young Lee, Jun Soo Kwon. Visualization: Seo Woo Kim. Writing_original draft: Seo Woo Kim. Writing_review \& editing: Minah Kim, Sun-Young Moon, Tae Young Lee, Jun Soo Kwon.

\section{ORCID iDs}

Seo Woo Kim

Sun-Young Moon

Wu Jeong Hwang

Silvia Kyungjin Lho

Sanghoon Oh

Tae Young Lee

Minah Kim

Jun Soo Kwon

https://orcid.org/0000-0001-8418-0210

https://orcid.org/0000-0001-7319-4131

https://orcid.org/0000-0002-2546-3103

https://orcid.org/0000-0001-7164-9358

https://orcid.org/0000-0002-7394-5211

https://orcid.org/0000-0002-0238-8147

https://orcid.org/0000-0001-8668-0817

https://orcid.org/0000-0002-1060-1462

\section{REFERENCES}

1. Burns T, Patrick D. Social functioning as an outcome measure in schizophrenia studies. Acta Psychiatr Scand 2007;116:403-418.

2. Cornblatt BA, Carrion RE, Addington J, Seidman L, Walker EF, Cannon TD, et al. Risk factors for psychosis: impaired social and role functioning. Schizophr Bull 2012;38:1247-1257.

3. Bora E, Yucel M, Pantelis C. Theory of mind impairment in schizophrenia: meta-analysis. Schizophr Res 2009;109:1-9.

4. Fett AKJ, Viechtbauer W, Dominguez MD, Penn DL, Van Os J, Krabbendam $\mathrm{L}$. The relationship between neurocognition and social cognition with functional outcomes in schizophrenia: a meta-analysis. Neurosci Biobehav Rev 2011;35:573-588.

5. Schaafsma SM, Pfaff DW, Spunt RP, Adolphs R. Deconstructing and reconstructing theory of mind. Trends Cogn Sci 2015;19:65-72.

6. Sprong M, Schothorst P, Vos E, Hox J, Van Engeland H. Theory of mind in schizophrenia - meta-analysis. Br J Psychiatry 2007;191:5-13.

7. Bentall RP, Fernyhough C, Morrison AP, Lewis S, Corcoran R. Prospects for a cognitive-developmental account of psychotic experiences. Br J Clin Psychol 2007;46:155-173.

8. Sabbagh MA, Moulson MC, Harkness KL. Neural correlates of mental state decoding in human adults: an event-related potential study. J Cogn Neurosci 2004;16:415-426.

9. Bora E, Eryavuz A, Kayahan B, Sungu G, Veznedaroglu B. Social functioning, theory of mind and neurocognition in outpatients with schizophrenia; mental state decoding may be a better predictor of social functioning than mental state reasoning. Psychiatry Res 2006;145:95-103.

10. Bora E, Pantelis C. Theory of mind impairments in first-episode psychosis, individuals at ultra-high risk for psychosis and in first-degree relatives of schizophrenia: systematic review and meta-analysis. Schizophr Res 2013;144:31-36

11. Lee TY, Hong SB, Shin NY, Kwon JS. Social cognitive functioning in prodromal psychosis: a meta-analysis. Schizophr Res 2015;164:28-34.

12. Zhang TH, Cui HR, Wei YY, Tang YY, Xu LH, Tang XC, et al. Progressive decline of cognition during the conversion from prodrome to psychosis with a characteristic pattern of the theory of mind compensated by neurocognition. Schizophr Res 2018;195:554-559.

13. Chung YS, Kang DH, Shin NY, Yoo SY, Kwon JS. Deficit of theory of mind in individuals at ultra-high-risk for schizophrenia. Schizophr 
Res 2008;99:111-118.

14. Hur JW, Byun MS, Shin NY, Shin YS, Kim SN, Jang JH, et al. General intellectual functioning as a buffer against theory-of-mind deficits in individuals at ultra-high risk for psychosis. Schizophr Res 2013;149: 83-87.

15. Baron-Cohen S, Wheelwright S, Hill J, Raste Y, Plumb I. The "Reading the Mind in the Eyes" test revised version: a study with normal adults, and adults with asperger syndrome or high-functioning autism. J Child Psychol Psychiatry 2001;42:241-251.

16. Blakemore SJ, Mills KL. Is adolescence a sensitive period for sociocultural processing? Annu Rev Psychol 2014;65:187-207.

17. Garcia NV, Scherf KS. Emerging sensitivity to socially complex expressions: a unique role for adolescence? Child Dev Perspect 2015;9:84-90.

18. Meinhardt-Injac B, Daum MM, Meinhardt G, Persike M. The twosystems account of theory of mind: testing the links to social- perceptual and cognitive abilities. Front Hum Neurosci 2018;12:25.

19. McGlade N, Behan C, Hayden J, O’Donoghue T, Peel R, Haq F, et al. Mental state decoding $\mathrm{v}$. mental state reasoning as a mediator between cognitive and social function in psychosis. Br J Psychiatry 2008;193: 77-78.

20. Schurz M, Radua J, Aichhorn M, Richlan F, Perner J. Fractionating theory of mind: a meta-analysis of functional brain imaging studies. Neurosci Biobehav Rev 2014;42:9-34.

21. Chung YS, Barch D, Strube M. A meta-analysis of mentalizing impairments in adults with schizophrenia and autism spectrum disorder. Schizophr Bull 2014;40:602-616.

22. Stanford AD, Messinger J, Malaspina D, Corcoran CM. Theory of mind in patients at clinical high risk for psychosis. Schizophr Res 2011;131:11-17.

23. Couture SM, Penn DL, Addington J, Woods SW, Perkins DO. Assessment of social judgments and complex mental states in the early phases of psychosis. Schizophr Res 2008;100:237-241.

24. Atkinson RJ, Fulham WR, Michie PT, Ward PB, Todd J, Stain H, et al. Electrophysiological, cognitive and clinical profiles of at-risk mental state: the longitudinal minds in transition (MinT) study. PLoS One 2017;12:e0171657.

25. Zhang TH, Tang YY, Cui HR, Lu X, Xu LH, Liu XH, et al. Theory of mind impairments in youth at clinical high risk of psychosis. Psychiatry Interpers Biol Process 2016;79:40-55.

26. Thompson AD, Bartholomeusz C, Yung AR. Social cognition deficits and the 'ultra high risk' for psychosis population: a review of literature. Early Interv Psychiatry 2011;5:192-202.

27. Dodell-Feder D, Ressler KJ, Germine LT. Social cognition or social class and culture? On the interpretation of differences in social cognitive performance. Psychol Med 2020;50:133-145.

28. Milligan K, Astington JW, Dack LA. Language and theory of mind: meta-analysis of the relation between language ability and false-belief understanding. Child Dev 2007;78:622-646.

29. Vetter NC, Leipold K, Kliegel M, Phillips LH, Altgassen M. Ongoing development of social cognition in adolescence. Child Neuropsychol 2013;19:615-629.

30. Kilford EJ, Garrett E, Blakemore SJ. The development of social cognition in adolescence: an integrated perspective. Neurosci Biobehav Rev 2016;70:106-120.

31. Ayesa-Arriola R, Setien-Suero E, Neergaard KD, Ferro A, Fatjo-Vilas M, Rios-Lago M, et al. Evidence for trait related theory of mind impairment in first episode psychosis patients and its relationship with processing speed: a 3 year follow-up study. Front Psychol 2016;7:592.

32. Guastella AJ, Hermens DF, Van Zwieten A, Naismith SL, Lee RS, Cacciotti-Saija C, et al. Social cognitive performance as a marker of positive psychotic symptoms in young people seeking help for mental health problems. Schizophr Res 2013;149:77-82.

33. Kwon JS, Byun MS, Lee TY, An SK. Early intervention in psychosis: insights from Korea. Asian J Psychiatry 2012;5:98-105.

34. Kim Z. Two-and four-subtest short forms of the Korean-Wechsler adult intelligence scale. Seoul J Psychiatry 1994;19:379-396.

35. Miller TJ, McGlashan TH, Rosen JL, Somjee L, Markovich PJ, Stein K, et al. Prospective diagnosis of the initial prodrome for schizophrenia based on the structured interview for prodromal syndromes: preliminary evidence of interrater reliability and predictive validity. Am J Psychiatry 2002;159:863-865.

36. Jung MH, Jang JH, Kang DH, Choi JS, Shin NY, Kim HS, et al. The reliability and validity of the Korean version of the structured interview for prodromal syndrome. Psychiatry Investig 2010;7:257-263.

37. Gardner DM, Murphy AL, O’Donnell H, Centorrino F, Baldessarini RJ. International consensus study of antipsychotic dosing. Am J Psychiatry 2010;167:686-693.

38. Lee HR, Nam G, Hur JW. Development and validation of the Korean version of the reading the mind in the eyes test. PLoS One 2020;15: e0238309.

39. Baker CA, Peterson E, Pulos S, Kirkland RA. Eyes and IQ: a metaanalysis of the relationship between intelligence and "Reading the Mind in the Eyes". Intelligence 2014;44:78-92.

40. Choudhury S, Blakemore SJ, Charman T. Social cognitive development during adolescence. Soc Cogn Affect Neurosci 2006;1:165-174.

41. Kimhy D, Corcoran C, Harkavy-Friedman JM, Ritzler B, Javitt DC, Malaspina D. Visual form perception: a comparison of individuals at high risk for psychosis, recent onset schizophrenia and chronic schizophrenia. Schizophr Res 2007;97:25-34.

42. Lee SY, Bang M, Kim KR, Lee MK, Park JY, Song YY, et al. Impaired facial emotion recognition in individuals at ultra-high risk for psychosis and with first-episode schizophrenia, and their associations with neurocognitive deficits and self-reported schizotypy. Schizophr Res 2015;165:60-65.

43. Vohs JL, Lysaker PH, Francis MM, Hamm J, Buck KD, Olesek K, et al. Metacognition, social cognition, and symptoms in patients with first episode and prolonged psychoses. Schizophr Res 2014;153:54-59. 